
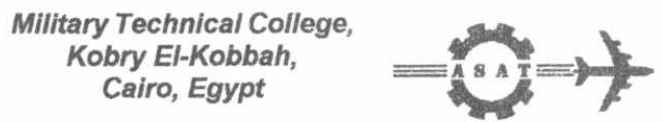

$9^{\text {th }}$ International Conference

On Aerospace Sciences \&

Aviation Technology

\title{
FLOW CHARACTERISTICS IN THE NEAR WAKE OF TANDEM CONES
}

\author{
$K^{K A D D A H}{ }^{\star}$ K. S., NOSIER* M. A., EL-BAZ ${ }^{\star \star}$ A. M. AND SELIM ${ }^{\star \star \star} M$.
}

\section{ABSTRACT}

Experimental measurements in the turbulent wake behind tandem open base cones with four different apex angles of 30, 45, 60 and 90 degrees and the separation distance between the cones fixed at 1.5 cone base diameter (1.5 D) at a Reynolds number of $4 \times 10^{4}$ were undertaken. Measurements in the wake of single cones at the same Reynolds number have also been performed for comparison. The measurements were extended up to a down stream distance of 10 diameters behind the second cone. All measurements were performed using hot wire anemometer with X-wire probe.

Mean velocity, turbulent intensity and shear stress show that the cone angle has an important effect on the wake development, drag force and mixing of the fluid in the near wake behind tandem cones. The measured drag coefficient increases with cone angle. The drag coefficient of tandem cones is lower than that of single open base cone of the same cone angle and Reynolds number. This is in accordance with the observed attenuation of turbulence in the wake of tandem cones.

\section{KEYWORDS}

Turbulence, Wakes, Cones.

Professor, Department of Mechanical Power Engineering, Ain Sahams University.

*t Lecturer, Department of Mechanical Power Engineering, Ain Sahams University.

Civil Aviation Institute, Imbaba, Giza. 


\title{
NOMENCLATURE
}

\author{
$C_{D} \quad$ Drag coefficient \\ d Cone base diameter \\ L Length of re-circulation zone \\ $r \quad$ Radial coordinate \\ $\mathrm{R} \quad$ Cone base radius \\ $\mathrm{R}_{1 / 2} \quad$ Half width of wake \\ $u_{\text {ms }}$ Root mean square of axial velocity fluctuations \\ U Mean axial velocity \\ $U_{\mathrm{C}} \quad$ Mean axial velocity at wake centerline \\ $\mathrm{U}_{\mathrm{e}} \quad$ Mean axial velocity in the free stream \\ uv Turbulent shear stress \\ $v_{\text {rms }}$ Root mean square of axial velocity fluctuations \\ $x \quad$ Axial distance measured from the upstream cone base \\ $x^{\prime} \quad$ Axial distance measured from downstrearn cone base
}

\section{INTRODUCTION}

Bluff bodies are widely used in many industrial applications. In combustors, for example, disks and cones are used as flame stabilizing devices. In heat exchangers, several shapes of bluff bodies have been employed in order to enhance mixing and to increase the heat transfer rates of internal passages. In either case the flow behind the bluff body is characterized by the presence of a recirculation zone of retarded flow. The apparent loss of the mean kinetic energy of the flow associated with this behavior is converted to turbulence in the vicinity of the bluff body. It is thus important to thoroughly understand the characteristics of flow behind bluff bodies in order to design such devices.

Numerous studies involving the flow behind single bluff body have been reported in the literature. Calvert[1] and Davies and Beer[2] reported measurements in the wake behind conical bodies of different cone angles and Reynolds number. Studies involving two or more bluff bodies, arranged in tandem, are seldom found in the available literature. Tatsutani et al [3] performed dye visualization experiments to investigate the effect of changing the separation distance between two cylinders on the flow behavior and heat transfer of the downstream cylinder. They investigated the flow for values of Reynolds number up to 1600 and for cylinder separation distance to cylinder diameter ratios between 0.25 and 4 . At a critical separation distance, they found the flow to become bi-stable and alternate between two patterns, i e. a closed inter cylinder gap recirculation and an oscillating wake formed behind the upstream cylinder. Valencia [4] performed a numerical study of the effect of the cylinder separation distance on the flow and heat transfer for three separation distance to diameter ratios of 1,2 , and 4 . $\mathrm{He}$ found that, for a separation distance to diarneter ratio of 4 , the largest heat transfer enhancement was achieved.

Liou and Chen [5] reported measurements of the flow past an array of bluff bodies aligned along a water tunnel axis using LDA. They used square ribs with 
separation distance to rib height ratio of 10 . The experimental measurements were compared with numerical computations using Reynolds stress turbulence model with several modifications.

The present study has two main objectives. The first is to obtain detailed information on the flow behaviour in the near wake region of tandem cones. The second objective is to highlight the differences between the flow characteristics behind the tandem cone configuration and those of single cones.

\section{EXPERIMENTAL SET-UP}

The experiments were carried out in a low turbulence, open-circuit wind tunnel with a square test section of $0.305 \times 0.305 \mathrm{~m}$ in area. The length of the test section in the flow direction is $0.60 \mathrm{~m}$. The turbulence intensity at the beginning of test section is less than $0.5 \%$. The air speed in the tunnel was fixed at $13.8 \mathrm{~m} / \mathrm{s}$ for all test cases.

Figure 1 shows a schematic drawing of the tandem cone configuration. The cones used have a $30 \mathrm{~mm}$ base diameter with cone angles of 30, 45, 60 and 90 degrees. The tandem cones were connected using a central rod $4 \mathrm{~mm}$ in diameter with a fixed separation distance between the cone bases of $4.5 \mathrm{~cm}$. The cones were positioned at the entrance of the test section using eight piano wires, $0.3 \mathrm{~mm}$ in diameter. The blockage ratio of the tunnel, expressed by the ratio of the model area to the test section area, was less than $1 \%$. Alignment checks were carried out during the initial setup of the experiment by measuring the mean velocity along two perpendicular planes at $\mathrm{x} / \mathrm{d}=10$.

The mean and fluctuating velocities in the wake were measured using a constant temperature hot wire anemometer [DANTEC 56C17] using X-wire probe. The sensing elements are $0.005-\mathrm{mm}$ diameter, Platinum-plated Tungsten wires. The number of samples collected from each channel of the X-wire probe was 4096. The hot wire signals were further analyzed using AQWIRE software of DANTEC [6] to calculate the moments of the velocity signals. The hot wire probe was calibrated using another low turbulence, variable speed wind tunnel. The ambient air flowing into this calibration tunnel passes through a calibrated nozzle. After measuring the static pressure difference between the exit section of the nozzle and the ambient, the air velocity at nozzle exit was calculated by applying Bernoulli's equation.

One of the disadvantages of using hot wire anemometer in measurements of recirculating flows is its inability to determine the direction of flow. In order to overcome this difficulty, a mirror imaging technique was used to obtain the mean velocity in the re-circulation zone behind the cones [1], [7], [8]. 


\section{PRESENTATION OF RESULTS}

\subsection{Mean Flow Measurements}

Figure 2 shows the measured variation of the centerline velocity difference $\left(U_{e}\right.$ $\left.\mathrm{U}_{\mathrm{c}}\right) / \mathrm{U}_{\mathrm{e}}$ with the axial distance $\mathrm{x}^{\prime} / \mathrm{d}$, for $90^{\circ}$ single open-base cone and tandem cones where $x^{\prime}$ is the downstream distance measured from the base of the second cone. The figure shows that the mean velocity deficit for tandem cones approach a $-2 / 3$ decay pattern faster than single open base cone.

Figure 3 shows the measured variation of wake half - width for the above cases. This half width is defined by the radial distance at which $50 \%$ of the mean velocity deficit occurs. There are some differences between the wake growth rates for tandem open base cones and the single cone of $90^{\circ}$. The spreading rate for tandem cones is slower than that for single cones.

Figure 4 shows the measured drag coefficient $C_{D}$ for tandem cones versus cone angle. This drag coefficient was calculated by applying the momentum integral equation at $x / d=10$. The results show that as the cone angle increases the drag coefficient increases in a linear relation. For comparison, Fig. 4 also includes the drag coefficients for single open base cones. As can be seen in the figure, $C_{D}$ for tandem open base cones is smaller than corresponding values for the single open base cones.

Figure 5 shows the variation of the normalized re-circulation zone length (L/d) with cone angle for closed base, open base, and tandem open base cones. This length is defined by the axial distance from the base of the front cone at which the axial velocity changes its sign from negative to positive. As can be seen in the figure, for single cones, L/d increases with cone angle. The figure also shows that the recirculation zone of tandem cones is generally larger than single cones, although it is nearly independent of cone angle.

\subsection{Turbulence Measurements}

Figure 6 shows the measured variation of normalized axial turbulence intensity $u_{r m s}$ at the centerline of the wake, with the axial distance $x^{\prime} / d$ for different cone angles of the tandem open base cones. The figure shows that as $x^{\prime} / d$ increases the turbulence intensity decreases at similar rates regardless of the cone angle. This figure also indicates that the axial turbulence intensity decay pattern follows a $-2 / 3$ decay law for $x^{\prime} / d>7$.

Figure 7 shows the variation of the radial turbulent intensity normalized by the corresponding axial intensity, along the centerline of the wake, for different cone angles. The figure shows that as cone angle increases the relative radial intensity increases and becomes close to a unity for most of the tested cones.

Figure 8 shows the variation of axial turbulence intensity, versus radial position for tandem open base cones at $x^{\prime} / d=10$ for different cone angles. The figure shows that the turbulence intensity increases as cone angle increases. 
For $90^{\circ}$ cones, an off axis peak at $r / R=1.5$ can be observed. For other cone angles the turbulent intensity profile is almost flat in the central part of the flow. Similar behavior was observed for the radial turbulent intensity, Fig. 9.

Figure 10 shows the variation of shear stress profiles at $x^{\prime} / d=10$ versus the radial position $\mathrm{r} / \mathrm{R}$ for different cone angles. The figure shows that the highest shear stress also correspond to cones of $90^{\circ}$ cones. The location of peak shear stress coincides with that of the axial turbulent intensity. The figure also indicates that tandem cones with $30^{\circ}$ cone angle, have the least shear stress and narrowest shear region, while the tandem cones with $90^{\circ}$ cone angles have the highest shear stress and a widest shear region.

\section{CONCLUSIONS}

1- Mean flow and turbulence properties for tandem cones approach asymptotic state faster than single cones.

2- Spreading of half width of tandem cone wake is slower than that of single cone of the same cone angle.

3 - Tandem cones experience less drag than single cones of the same cone angle.

4- Intensity of turbulence increases with cone angle. The highest levels of all components of turbulence were observed for $90^{\circ}$ tandem cones. 


\section{REFERENCES}

1. Calvert, J. R., "Experiment on the low speed flow past cones", J. Fluid Mechanics, Vol. 27, Part 2, pp 273-2:89, 1967.

2. Davies, T. W. and Beer, J. M., "Flow in the wake of bluff body flame stabilizers", $13^{\text {th }}$ Symposium (International) on Combustion, pp 631638, The Combustion institute, 1971 .

3. Tatsutani, K., Devarakonda, R. and Humphrey, J. A., "Unsteady flow and heat transfer for cylinder pairs in a channel", Int $\mathrm{J}$. Heat and Mass Transfer, vol 36, $n^{\circ} .13$, pp 3311-332.8, 1993.

4. Valencia, A., "Unsteady flow and heat transfer in a channel with a built-in tandem of rectangular cylinders', Numerical heat transfer, part A, vol 29, pp 613-623, 1996

5. Liou, T. M. and Chen, S. H. "Turbulent flow past an array of bluff bodies aligned along the channel axis", J. Fluids Eng, vol 120, pp 520-530, 1998

6. DANTEC Electronics Inc. ACQWIRE. Technical Reference Manual, DANTEC, 1981

7. Selim, M. A., "Structure of turbulent wakes behind bluff bodies", PhD Thesis, Ain Shams University, Cairo, 1997.

8. Kaddah, K. S., Nosier M. A., El-Baz. A. M., Selim M. A. "Turbulent flow in the near wake of twin disks". Eng. Res. Jour. vol 62, pp 146160, April 1999, Helwan University, Mataria, Cairo. 


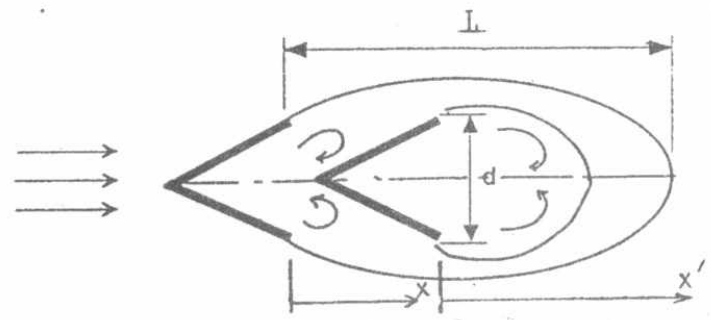

Fig. 1 Configuration of tandem cones

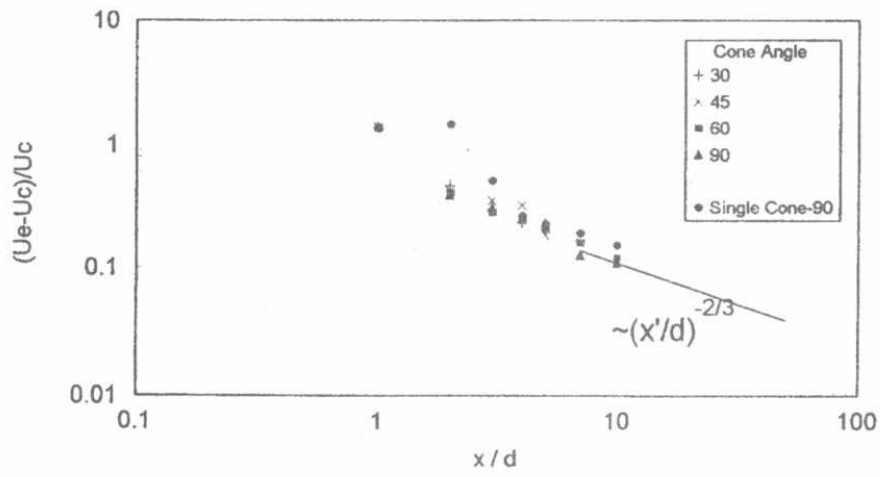

Fig. 2 Variation of axial mean velocity defect for tandem cones 


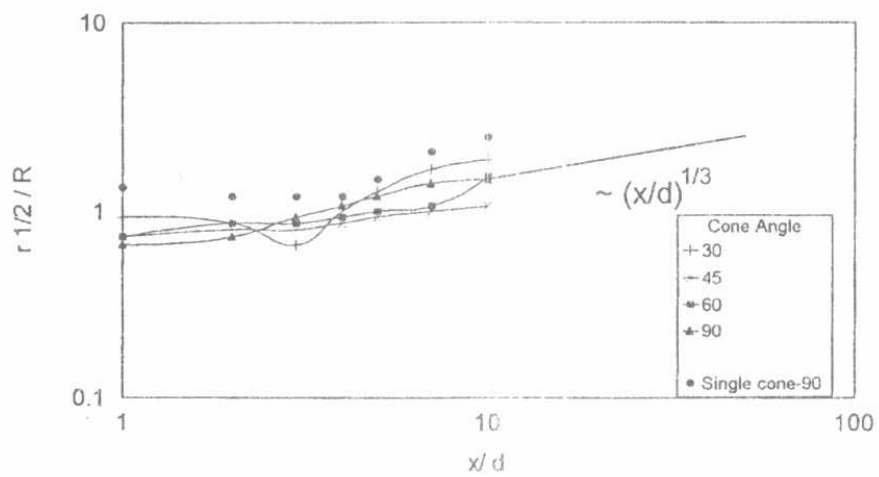

Fig. 3 Variation of wake half width for tandem cones.

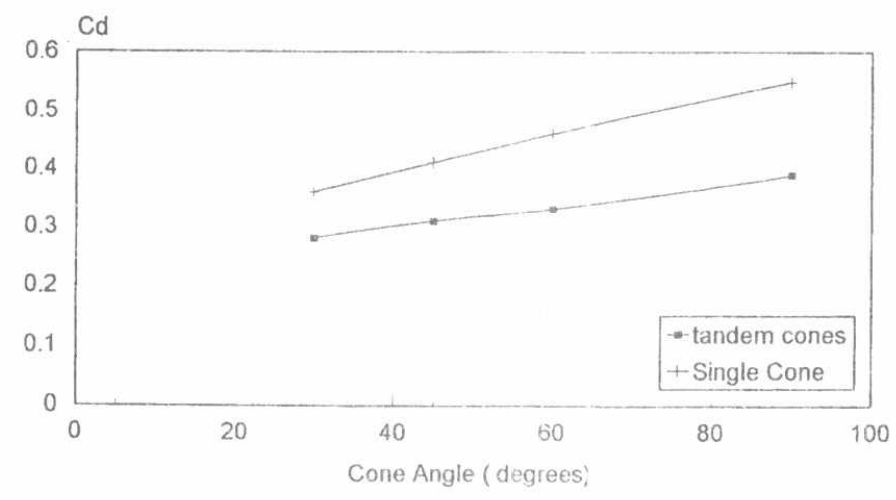

Fig (4) variation of $\mathrm{Cd}$ for tandem cones. 


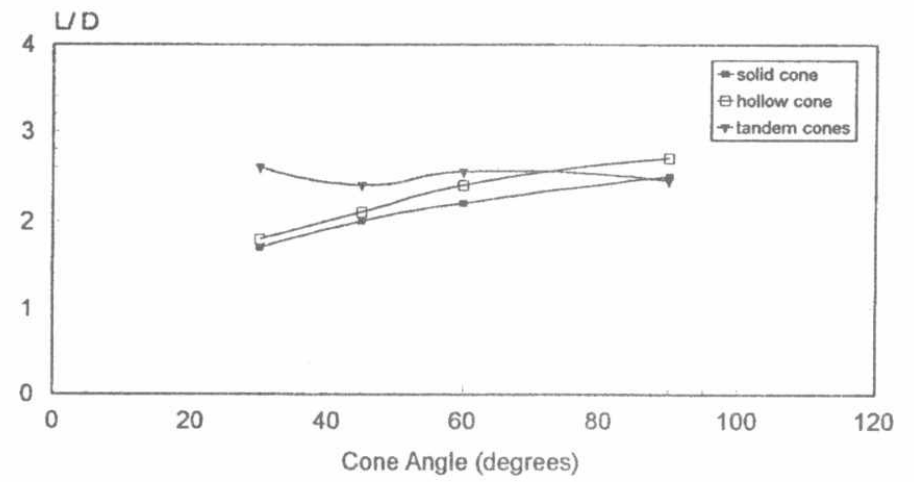

Fig. 5 Variation of recirculation zone length for cones.

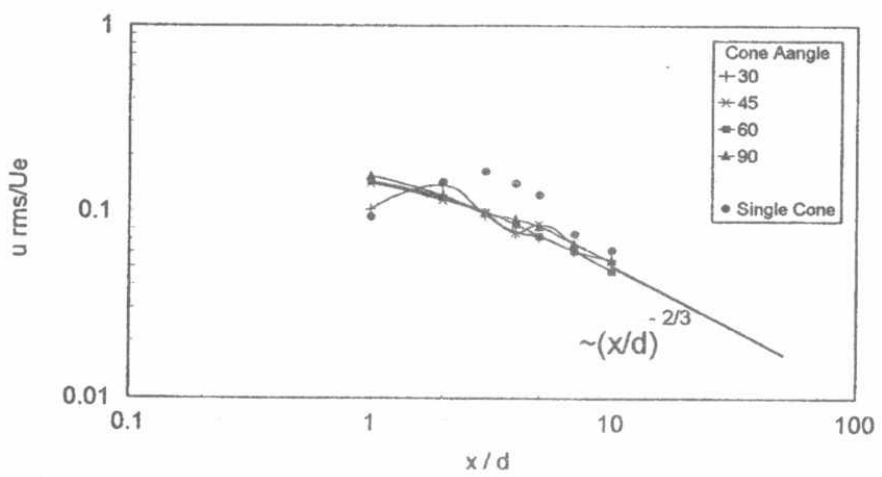

Fig. 6 Variation of axial turbulence intensity for tandem cones 


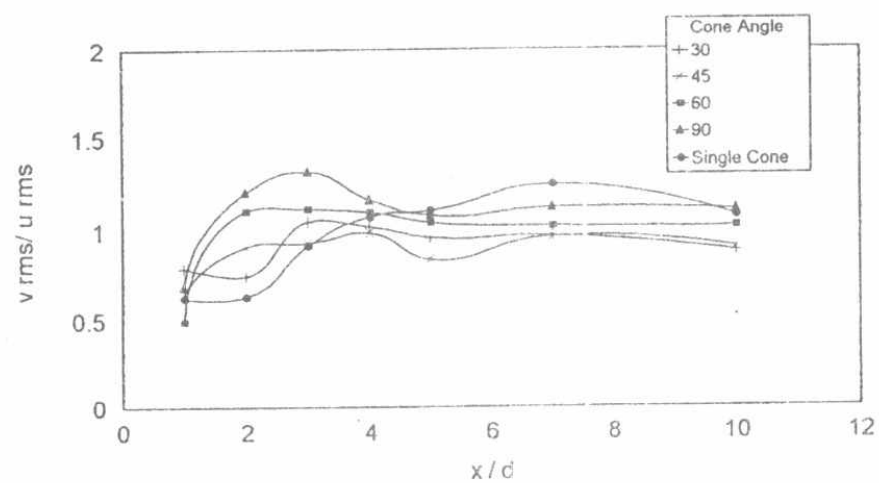

Fig. 7 Variation of relative radial turbulence intensity for tandem cones

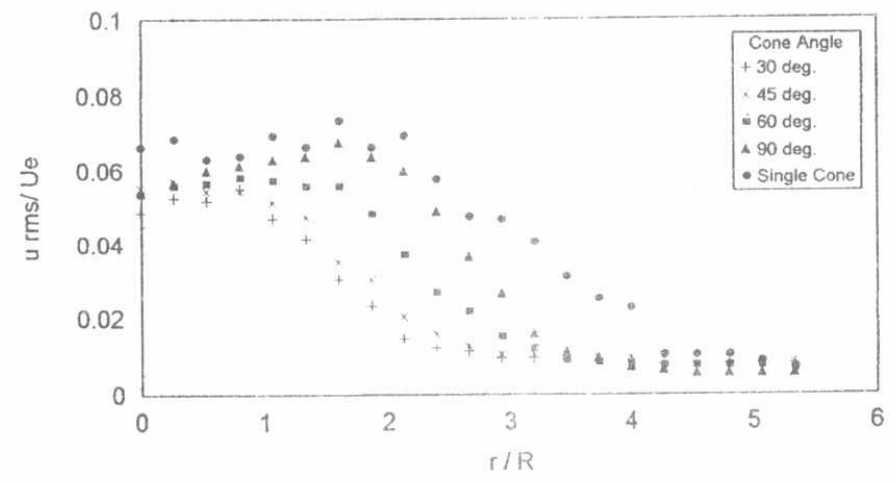

Fig. 8 Variation of axial turbulence intensity for tandem cones at $x / d=10$ 


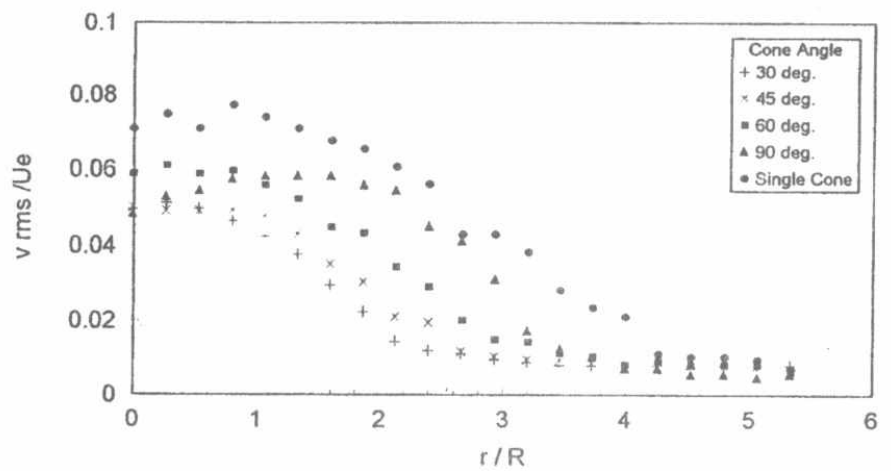

Fig. 9 Variation of radial turbulence intensity for tandem cones at $\mathrm{x} / \mathrm{d}=10$

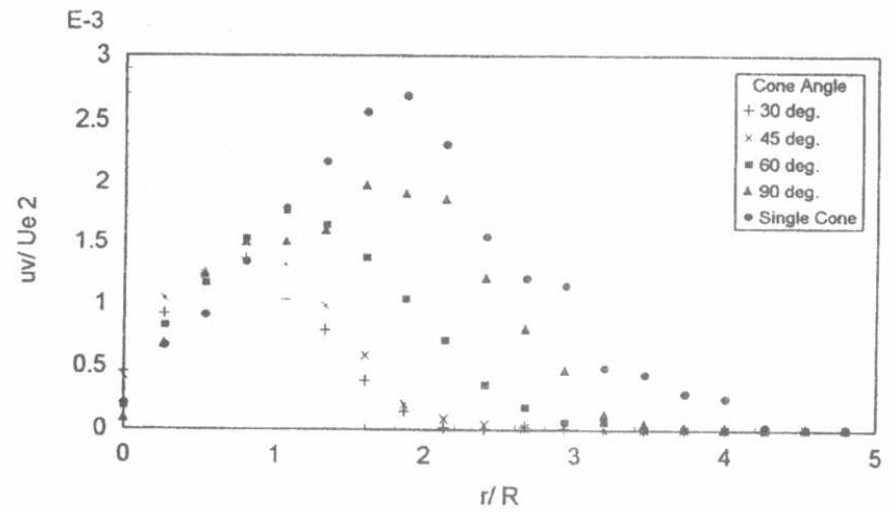

Fig. 10 Variation of shear stress for tandem cones at $x / d=10$ 